\title{
Garcinia cambogia Extract
}

National Cancer Institute

\section{Source}

National Cancer Institute. Garcinia cambogia Extract. NCI Thesaurus. Code C89786.

An extract from the fruit of the Garcinia cambogia. It contains hydroxycitric acid and is claimed to be an appetite suppressant. 